\title{
JOINT DISTRIBUTION \\ IN RESIDUE CLASSES OF THE BASE- $q$ AND OSTROWSKI DIGITAL SUMS
}

\author{
Divyum SHARMA
}

Department of Pure Mathematics, University of Waterloo, Ontario, CANADA

ABSTRACT. Let $q$ be an integer greater than or equal to 2 , and let $S_{q}(n)$ denote the sum of digits of $n$ in base $q$. For

$$
\alpha=[0 ; \overline{1, m}], m \geq 2,
$$

let $S_{\alpha}(n)$ denote the sum of digits in the Ostrowski $\alpha$-representation of $n$. Let $m_{1}, m_{2} \geq 2$ be integers with

$$
\operatorname{gcd}\left(q-1, m_{1}\right)=\operatorname{gcd}\left(m, m_{2}\right)=1 .
$$

We prove that there exists $\delta>0$ such that for all integers $r_{1}, r_{2}$,

$$
\begin{aligned}
& \left|\left\{0 \leq n<N: S_{q}(n) \equiv r_{1}\left(\bmod m_{1}\right), S_{\alpha}(n) \equiv r_{2}\left(\bmod m_{2}\right)\right\}\right| \\
& =\frac{N}{m_{1} m_{2}}+O\left(N^{1-\delta}\right) .
\end{aligned}
$$

The asymptotic relation implied by this equality was proved by Coquet, Rhin \& Toffin and the equality was proved for the case $\alpha=[\overline{1}]$ by Spiegelhofer.

\section{Communicated by Werner Georg Nowak}

\section{Introduction}

Let $q$ be an integer greater than or equal to 2 , and let $S_{q}(n)$ denote the sum of digits of $n$ in base $q$. Much effort has been made to understand the behaviour of this function. Bush [9] studied the asymptotic behaviour of its mean value. The distribution of the values of this function has also been investigated [15]. There has been a keen interest in the sum of digits of primes [18] and polynomials [4]. (We also refer to [5], 12] and the references there for other related results.)

(C) 2019 BOKU-University of Natural Resources and Life Sciences and Mathematical Institute, Slovak Academy of Sciences.

2010 Mathematics Subject Classification: 11N64, 11N69, $11 \mathrm{~A} 63$.

Keyw ords: Ostrowski representation, Sum of digits function, Joint distribution.

Licensed under the Creative Commons Attribution-NC-ND 4.0 International Public License. 
Throughout this paper, $q, q_{1}, q_{2}, m_{1}, m_{2}$ denote integers greater than or equal to 2. Gel'fond [13] proved that if $m_{1}$ is coprime to $q-1$, then the function $S_{q}(n)$ is uniformly distributed modulo $m_{1}$. Further, he conjectured that if

$$
\operatorname{gcd}\left(q_{1}, q_{2}\right)=\operatorname{gcd}\left(m_{1}, q_{1}-1\right)=\operatorname{gcd}\left(m_{2}, q_{2}-1\right)=1,
$$

then there exists $\delta=\delta\left(q_{1}, q_{2}, m_{1}, m_{2}\right)>0$ such that

$$
\begin{aligned}
& \left|\left\{0 \leq n<N: S_{q_{1}}(n) \equiv r_{1}\left(\bmod m_{1}\right), S_{q_{2}}(n) \equiv r_{2}\left(\bmod m_{2}\right)\right\}\right| \\
& =\frac{N}{m_{1} m_{2}}+O\left(N^{1-\delta}\right)
\end{aligned}
$$

for all integers $r_{1}, r_{2}$. The asymptotic relation implied in this conjecture was proved by Bésineau [7]; while the conjecture was proved in its full strength by Kim [16] (See also [5] for some recent improvements of Kim's result).

In this paper we are concerned with the above problem for the sum of digits functions of the base- $q$ and Ostrowski representation of integers. In 1922, Ostrowski [19] discovered a numeration system based on continued fractions. He showed that the sequence of the denominators of the convergents to the simple continued fraction expansion of an irrational number forms the basis for a numeration system. More precisely, he proved the following result.

Theorem 1.1. [1, Theorem 3.9.1] Let $\alpha$ be an irrational real number having continued fraction expansion $\left[a_{0} ; a_{1}, \ldots\right]$. Let $\left(q_{i}\right)_{i \geq 0}$ be the sequence of the denominators of the convergents to the continued fraction expansion. Then every non-negative integer $n$ can be expressed uniquely as

$$
n=\sum_{0 \leq i \leq \ell} b_{i} q_{i}
$$

where the $b_{i}$ 's are integers satisfying

(i) $0 \leq b_{0}<a_{1}$.

(ii) $0 \leq b_{i} \leq a_{i+1}$ for $i \geq 1$.

(iii) For $i \geq 1$, if $b_{i}=a_{i+1}$, then $b_{i-1}=0$.

In fact, the three conditions above are equivalent to the inequality

$$
b_{0} q_{0}+b_{1} q_{1}+\cdots+b_{i} q_{i}<q_{i+1} .
$$

Note that Condition (iii) states that the relation $q_{i+1}=a_{i+1} q_{i}+q_{i-1}$ cannot be used to replace a linear combination of summands with another summand. The expression given in (1) is called the Ostrowski $\alpha$-representation of $n$. 


\section{BASE- $q$ AND OSTROWSKI SUM-OF-DIGITS FUNCTIONS}

See [6] for a survey on the connections between the Ostrowski numeration systems and combinatorics of words, and [3] for a study of ergodic and topologicaldynamical properties of various dynamical systems associated to Ostrowski $\alpha$ -representations. We refer to [8] for an analysis of the asymptotic average of the number of non-zero terms required in these representations.

If the Ostrowski $\alpha$-representation of a positive integer $n$ is given by

$$
n=\sum_{0 \leq i \leq \ell} b_{i}(n) q_{i},
$$

let

$$
S_{\alpha}(n)=\sum_{0 \leq i \leq \ell} b_{i}(n)
$$

be the sum of digits. In [10], Coquet, Rhin \& Toffin studied the relation between the functions $S_{q}(n)$ and $S_{\alpha}(n)$. They proved the following theorem.

Theorem. Let $q$ be an integer greater than or equal to 2 and let $\alpha$ be an irrational real number. The sequence $n \rightarrow x S_{q}(n)+y S_{\alpha}(n)$ is uniformly distributed modulo 1 if and only if at least one of $x$ and $y$ is irrational.

In [20], Spiegelhofer considered the case when

$$
\alpha=\frac{1+\sqrt{5}}{2}=[\overline{1}] .
$$

(Note that in this case, the sequence $\left(q_{i}\right)$ is the sequence of Fibonacci numbers and that every non-negative integer can be uniquely expressed as a sum of nonconsecutive Fibonacci numbers. This representation is known as the Zeckendorf representation of integers (see [23]).) He proved that if $\theta \in \mathbb{R}$ and $\gamma \in \mathbb{R} \backslash \mathbb{Z}$, then

$$
\sum_{n<N} e\left(\theta S_{q}(n)+\gamma S_{\alpha}(n)\right)=O\left(N^{1-\delta}\right)
$$

for some $\delta>0$. (Throughout this paper, $e(x)$ denotes $\exp (2 \pi i x)$.) As a consequence, he obtained

Theorem. [20, Corollary 5.3] Let $\alpha=(1+\sqrt{5}) / 2$ and let $q, m_{1}, m_{2} \geq 2$ be integers with $\operatorname{gcd}\left(m_{1}, q-1\right)=1$. There exists $\delta>0$ such that for all integers $r_{1}, r_{2}$,

$$
\begin{aligned}
& \left|\left\{0 \leq n<N: S_{q}(n) \equiv r_{1}\left(\bmod m_{1}\right), S_{\alpha}(n) \equiv r_{2}\left(\bmod m_{2}\right)\right\}\right| \\
& =\frac{N}{m_{1} m_{2}}+O\left(N^{1-\delta}\right) .
\end{aligned}
$$


In [11, Coquet, Rhin \& Toffin gave three sufficient conditions for the set

$$
\left\{n \in \mathbb{N}: S_{q}(n) \equiv r_{1}\left(\bmod m_{1}\right), S_{\alpha}(n) \equiv r_{2}\left(\bmod m_{2}\right)\right\}
$$

to have asymptotic density equal to $1 /\left(m_{1} m_{2}\right)$. One of these conditions is that the sequence $\left(q_{k}\right)$ be lacunary and $\operatorname{gcd}\left(a_{k}, m_{2}\right)$ be equal to one for infinitely many indices $k$. Note that this condition is satisfied for

$$
\alpha=[0 ; \overline{1, m}]=\frac{-m+\sqrt{m^{2}+4 m}}{2}, m \geq 2
$$

as

$$
\frac{q_{k+1}}{q_{k}} \geq \begin{cases}1+\frac{q_{k-1}}{q_{k}} \geq 1+\frac{1}{m+1} & \text { if } a_{k+1}=1 \\ m+\frac{q_{k-1}}{q_{k}} \geq m+\frac{1}{2} & \text { if } a_{k+1}=m .\end{cases}
$$

For these values of $\alpha$, we improve the above asymptotic estimate to an estimate with error term $O\left(N^{1-\delta}\right)$. Let $\|x\|=\min _{j \in \mathbb{Z}}|x-j|$. We prove

Theorem 1.2. Let $q \geq 2$ be an integer and let

$$
\alpha=[0 ; \overline{1, m}, m \geq 2 .
$$

Let $\theta, \gamma \in \mathbb{R}$ with $\|m \gamma\| \neq 0$. Then there exists $\delta>0$ such that

$$
\sum_{n<N} e\left(\theta S_{q}(n)+\gamma S_{\alpha}(n)\right)=O\left(N^{1-\delta}\right) .
$$

As a consequence, we obtain

Corollary 1.3. Let $q$ and $\alpha$ be as in Theorem 1.2 and let $m_{1}, m_{2} \geq 2$ be integers with $\operatorname{gcd}\left(q-1, m_{1}\right)=\operatorname{gcd}\left(m, m_{2}\right)=1$. There exists $\delta>0$ such that for all integers $r_{1}, r_{2}$,

$$
\begin{aligned}
\mid\left\{0 \leq n<N: S_{q}(n)\right. & \left.\equiv r_{1}\left(\bmod m_{1}\right), S_{\alpha}(n) \equiv r_{2}\left(\bmod m_{2}\right)\right\} \mid \\
& =\frac{N}{m_{1} m_{2}}+O\left(N^{1-\delta}\right) .
\end{aligned}
$$

The proof relies on Weyl's and van der Corput's method. In Section 2, we introduce some notation and record some preliminary lemmas. Following [20], we obtain a characterization of integers with the same initial digits in their Ostrowski $\alpha$-representations in Section 3. We then use it to obtain an analogue of inverse discrete Fourier transform in this case and also derive a uniform upper bound for the Fourier coefficients. Finally, we prove Theorem 1.2 and Corollary 1.3 in Section 4. 


\section{BASE- $q$ AND OSTROWSKI SUM-OF-DIGITS FUNCTIONS}

\section{Preliminaries}

Let $q, m, \alpha, \theta, \gamma$ be as in the statement of Theorem 1.2. Let $\lceil x\rceil$ denote the smallest integer greater than or equal to $x$ and $\{x\}$ denote the fractional part of $x$. Recall that the Vinogradov symbol $f \ll F$ means that there is a constant $c$ such that the inequality $|f| \leq c F$ holds. The implied constant $c$ can depend on $q, m, \gamma$. We will repeatedly use the fact that for real numbers $x, y$ and a positive integer $a$, we have $\|x+y\| \leq\|x\|+\|y\|$ and hence $\|a x\| \leq a\|x\|$.

Since

$$
\alpha=[0 ; \overline{1, m}],
$$

we have $q_{0}=q_{1}=1$ and

$$
q_{i}= \begin{cases}m q_{i-1}+q_{i-2} & \text { if } i \text { is even } \\ q_{i-1}+q_{i-2} & \text { if } i \text { is odd }\end{cases}
$$

Let $d=m^{2}+4 m$ and

$$
\varphi=\frac{m+2+\sqrt{d}}{2} .
$$

Then

$$
\begin{aligned}
& q_{2 \ell}=\frac{m+\sqrt{d}}{2 \sqrt{d}} \varphi^{\ell}-\frac{m-\sqrt{d}}{2 \sqrt{d}} \varphi^{-\ell}, \\
& q_{2 \ell+1}=\frac{1}{\sqrt{d}} \varphi^{\ell+1}-\frac{1}{\sqrt{d}} \varphi^{-\ell-1} .
\end{aligned}
$$

By Theorem 1.1, the digits in the $\alpha$-representation (3) of a positive integer $n$ satisfy

$$
b_{2 \ell}(n) \leq 1 \text { and } b_{2 \ell+1}(n) \leq m
$$

for all non-negative integers $\ell$. Given an integer $k \geq 1$, let $t_{\alpha}(n ; k)$ denote the truncation of the sum in (3) after $k$ digits, i.e.,

$$
t_{\alpha}(n ; k)=\sum_{0 \leq i \leq k-1} b_{i}(n) q_{i}
$$

and let $S_{\alpha, k}(n)$ denote the sum of the first $k$ digits, i.e.,

$$
S_{\alpha, k}(n)=\sum_{0 \leq i \leq k-1} b_{i}(n) .
$$

By (2),

$$
t_{\alpha}(n ; k)<q_{k} .
$$

Next, let

$$
S_{q, t}(n)=S_{q}\left(n\left(\bmod q^{t}\right)\right) .
$$


Let $G_{t}(\ell)=G_{t}(\ell, \theta)$ denote the discrete Fourier coefficients of the function $e\left(\theta S_{q}(n)\right)$, i.e.,

$$
G_{t}(\ell, \theta)=\frac{1}{q^{t}} \sum_{u<q^{t}} e\left(\theta S_{q}(u)-\ell u q^{-t}\right)
$$

Then

$$
\begin{aligned}
e\left(\theta S_{q, t}(n)\right) & =\sum_{\ell<q^{t}} e\left(\ell n q^{-t}\right) G_{t}(\ell, \theta), \\
e\left(-\theta S_{q, t}(n)\right) & =\sum_{\ell<q^{t}} e\left(\ell n q^{-t}\right) \overline{G_{t}(-\ell, \theta)} .
\end{aligned}
$$

Note that, by Parseval's identity,

$$
\sum_{\ell<q^{t}}\left|G_{t}(\ell)\right|^{2}=1
$$

For negative integers $n$, we define

$$
S_{q}(n)=S_{\alpha}(n)=0 .
$$

We now list some results needed in the proof. The following is an elementary lemma on exponential sums.

Lemma 2.1. Let $x \in \mathbb{R}$ and $N, R \geq 0$. Then

(i) [17, Lemma1]

$$
\left|\sum_{n<N} e(n x)\right| \leq \min \left(N, \frac{1}{2\|x\|}\right)
$$

(ii)

$$
\sum_{|r|<R}(R-|r|) e(r x)=\left|\sum_{r<R} e(r x)\right|^{2}
$$

We now record an estimate from [20], which is proved using a discrepancy estimate for the sequence $(n \varphi)$, where $\varphi$ has bounded partial quotients. We use it for $\varphi$ as given in (4).

Lemma 2.2. 20, Lemma 5.8] Let $I$ be a finite interval in $\mathbb{Z}$. Let $K$ and a be real numbers with $K \geq 1$. Then

$$
\sum_{h \in I} \min \left(K, \frac{1}{\|a+h \varphi\|^{2}}\right) \ll \sqrt{K} \lambda(I)+K \ln \lambda(I) .
$$

(Here, $\lambda$ denotes the Lebesgue measure on $\mathbb{R}$.) 


\section{BASE- $q$ AND OSTROWSKI SUM-OF-DIGITS FUNCTIONS}

Next, we state the version of the Weyl-van der Corput inequality that will be used later.

Lemma 2.3. [14, Lemma 2.5] Let $z_{0}, \ldots, z_{N-1}$ be complex numbers. For all positive integers $R$, we have

$$
\left|\sum_{n=0}^{N-1} z_{n}\right|^{2} \leq \frac{N+R}{R} \sum_{|r|<R}\left(1-\frac{|r|}{R}\right)\left|\sum_{\substack{0 \leq n<N \\ 0 \leq n+r<N}} \bar{z}_{n} z_{n+r}\right| .
$$

The following lemma states that for most integers $n$, the representations of $n$ and $n+r$ may differ at digits corresponding to the first few base elements only.

Lemma 2.4. Let $N, r, k, t$ be non-negative integers with $k \geq 2$ and let $\theta, \gamma$ be real numbers. Then

$$
\begin{aligned}
& \text { (i) } \quad\left|\left\{n<N: e\left(\theta S_{q}(n+r)\right) \overline{e\left(\theta S_{q}(n)\right)} \neq e\left(\theta S_{q, t}(n+r)\right) \overline{e\left(\theta S_{q, t}(n)\right)}\right\}\right| \\
& \leq \frac{N r}{q^{t}}+r . \\
& \text { (ii) }\left|\left\{n<N: e\left(\gamma S_{\alpha}(n+r)\right) \overline{e\left(\gamma S_{\alpha}(n)\right)} \neq e\left(\gamma S_{\alpha, k}(n+r)\right) \overline{e\left(\gamma S_{\alpha, k}(n)\right)}\right\}\right| \\
& \leq \frac{N r}{q_{k-1}} .
\end{aligned}
$$

Proof.

See [20, Lemma 1.17] for a proof of (i) and [21, Lemma 2.6] for a proof of (ii).

\section{Lemmas}

We first derive a characterization (Corollary 3.3) of integers $n$ with the same value of $t_{\alpha}(n ; k)$, for a given $k$. Later, we use this to obtain the discrete Fourier transform for the function $e\left(\gamma S_{\alpha, k}(n)\right)$. This is analogous to [20, Proposition 5.7 \& Proposition 5.4].

LEMma 3.1. Let $k \geq 2$ be an integer. Let $p_{k}(n)=(-1)^{k} n \varphi$, where $\varphi$ is as in (41). Define

$$
A_{k}^{(1)}= \begin{cases}{\left[\frac{m-\sqrt{d}}{2 \varphi^{k_{0}}}, \frac{1}{\varphi^{k_{0}}}\right)} & \text { if } k=2 k_{0}, \quad k_{0} \in \mathbb{N}, \\ {\left[\frac{-1}{\varphi^{k_{0}+1}}, \frac{-m+\sqrt{d}}{2 \varphi^{k} 0}\right)} & \text { if } k=2 k_{0}+1, k_{0} \in \mathbb{N},\end{cases}
$$




$$
A_{k}^{(2)}= \begin{cases}{\left[\frac{m-\sqrt{d}}{2 \varphi^{k}}, \frac{1}{\varphi^{k_{0}+1}}\right)} & \text { if } k=2 k_{0}, \quad k_{0} \in \mathbb{N}, \\ {\left[\frac{-1}{\varphi^{k_{0}+1}}, \frac{-m-1+\sqrt{d}}{\varphi^{k}}\right)} & \text { if } k=2 k_{0}+1, k_{0} \in \mathbb{N},\end{cases}
$$

and

$$
R_{k}(u)=p_{k}(u)+ \begin{cases}A_{k}^{(1)} & \text { if } 0 \leq u<q_{k-1} \\ A_{k}^{(2)} & \text { if } q_{k-1} \leq u<q_{k}\end{cases}
$$

Then

$$
p_{k}(n) \in R_{k}\left(t_{\alpha}(n ; k)\right)+\mathbb{Z}
$$

Proof. By (5) and the definition of $t_{\alpha}(n ; k)$,

$$
\begin{aligned}
& n \varphi-t_{\alpha}(n ; k) \varphi \\
& =\sum_{\ell \geq\lceil k / 2\rceil} b_{2 \ell}(n) \varphi\left(\frac{m+\sqrt{d}}{2 \sqrt{d}} \varphi^{\ell}-\frac{m-\sqrt{d}}{2 \sqrt{d}} \varphi^{-\ell}\right) \\
& +\sum_{\ell \geq\lceil(k-1) / 2\rceil} b_{2 \ell+1}(n) \varphi\left(\frac{\varphi^{\ell+1}}{\sqrt{d}}-\frac{\varphi^{-\ell-1}}{\sqrt{d}}\right) \\
& =\sum_{\ell \geq\lceil k / 2\rceil} b_{2 \ell}(n) q_{2 \ell+2} \\
& +\frac{m-\sqrt{d}}{2 \sqrt{d}} \sum_{\ell \geq\lceil k / 2\rceil} b_{2 \ell}(n)\left(\varphi^{-\ell-1}-\varphi^{-\ell+1}\right) \\
& +\sum_{\ell \geq\lceil(k-1) / 2\rceil} b_{2 \ell+1}(n) q_{2 \ell+3} \\
& +\frac{1}{\sqrt{d}} \sum_{\ell \geq\lceil(k-1) / 2\rceil} b_{2 \ell+1}(n)\left(\varphi^{-\ell-2}-\varphi^{-\ell}\right) \\
& =\sum_{\ell \geq\lceil k / 2\rceil} b_{2 \ell}(n) q_{2 \ell+2} \\
& +\sum_{\ell \geq\lceil(k-1) / 2\rceil} b_{2 \ell+1}(n) q_{2 \ell+3} \\
& +\frac{(-m+\sqrt{d})}{2} \sum_{\ell \geq\lceil k / 2\rceil} \frac{b_{2 \ell}(n)}{\varphi^{\ell}}-\sum_{\ell \geq\lceil(k-1) / 2\rceil} \frac{b_{2 \ell+1}(n)}{\varphi^{\ell+1}} .
\end{aligned}
$$

Note that the first two terms in the above expression are integers. 


\section{BASE- $q$ AND OSTROWSKI SUM-OF-DIGITS FUNCTIONS}

We first consider the case when $k$ is even. Write $k=2 k_{0}, k_{0} \in \mathbb{N}$. Now

$$
\sum_{\ell \geq\lceil(k-1) / 2\rceil} \frac{b_{2 \ell+1}(n)}{\varphi^{\ell+1}} \leq m \sum_{\ell \geq k_{0}} \frac{1}{\varphi^{\ell+1}}=\frac{-m+\sqrt{d}}{2 \varphi^{k_{0}}}
$$

since

$$
\frac{1}{1-\varphi^{-1}}=\frac{2}{-m+\sqrt{d}}
$$

Suppose that $b_{k-1}(n)=0$. Then

$$
\frac{(-m+\sqrt{d})}{2} \sum_{\ell \geq\lceil k / 2\rceil} \frac{b_{2 \ell}(n)}{\varphi^{\ell}} \leq \frac{(-m+\sqrt{d})}{2} \sum_{\ell \geq k_{0}} \frac{1}{\varphi^{\ell}}=\frac{1}{\varphi^{k_{0}}} .
$$

If $b_{k-1}(n) \neq 0$, then by condition (iii) of Theorem 1.1, $b_{k}(n) \neq 1$. Hence $b_{k}(n)=0$ and

$$
\frac{(-m+\sqrt{d})}{2} \sum_{\ell \geq\lceil k / 2\rceil} \frac{b_{2 \ell}(n)}{\varphi^{\ell}} \leq \frac{(-m+\sqrt{d})}{2} \sum_{\ell \geq k_{0}+1} \frac{1}{\varphi^{\ell}}=\frac{1}{\varphi^{k_{0}+1}} .
$$

Further, note that if $b_{k-1}(n)=0$, then $t_{\alpha}(n ; k)=t_{\alpha}(n ; k-1)$ and hence by (6),

$$
t_{\alpha}(n ; k)=t_{\alpha}(n ; k-1)<q_{k-1} .
$$

If $b_{k-1}(n) \neq 0$, then

$$
t_{\alpha}(n ; k)=\sum_{0 \leq i \leq k-1} b_{i}(n) q_{i} \geq q_{k-1} .
$$

This proves the lemma when $k$ is even. Next, we consider the case when $k$ is odd. Write $k=2 k_{0}+1, k_{0} \in \mathbb{N}$. Then

$$
\begin{aligned}
& n(-\varphi)-t_{\alpha}(n ; k)(-\varphi) \\
& \equiv \frac{(m-\sqrt{d})}{2} \sum_{\ell \geq k_{0}+1} \frac{b_{2 \ell}(n)}{\varphi^{\ell}}+\sum_{\ell \geq k_{0}} \frac{b_{2 \ell+1}(n)}{\varphi^{\ell+1}}(\bmod 1) .
\end{aligned}
$$

Since

we get

$$
\sum_{\ell \geq k_{0}+1} \frac{b_{2 \ell}(n)}{\varphi^{\ell}} \leq \frac{1}{\varphi^{k_{0}+1}} \frac{2}{-m+\sqrt{d}}
$$

$$
\frac{(m-\sqrt{d})}{2} \sum_{\ell \geq k_{0}+1} \frac{b_{2 \ell}(n)}{\varphi^{\ell}} \geq-\frac{1}{\varphi^{k_{0}+1}} .
$$

Suppose that $b_{k-1}(n)=0$. Then

$$
\sum_{\ell \geq k_{0}} \frac{b_{2 \ell+1}(n)}{\varphi^{\ell+1}} \leq \frac{m}{\varphi^{k_{0}+1}} \frac{2}{-m+\sqrt{d}}=\frac{-m+\sqrt{d}}{2 \varphi^{k_{0}}} .
$$


If $b_{k-1}(n) \neq 0$, then by condition (iii) of Theorem 1.1, $b_{k}(n) \neq m$. Hence

$$
\begin{aligned}
\sum_{\ell \geq k_{0}} \frac{b_{2 \ell+1}(n)}{\varphi^{\ell+1}} & \leq \frac{m-1}{\varphi^{k_{0}+1}}+m \sum_{\ell \geq k_{0}+1} \frac{1}{\varphi^{\ell+1}} \\
& =\frac{m-1}{\varphi^{k_{0}+1}}+\frac{-m+\sqrt{d}}{2 \varphi^{k_{0}+1}} \\
& =\frac{-m-1+\sqrt{d}}{\varphi^{k_{0}}} .
\end{aligned}
$$

As before, this proves the lemma when $k$ is odd.

Lemma 3.2. Fix an integer $k \geq 2$. The sets

$$
R_{k}(u)+\mathbb{Z}, 0 \leq u<q_{k},
$$

form a partition of $\mathbb{R}$.

Proof. For each integer $u$ with $0 \leq u<q_{k}$, let

$$
\tilde{R}_{k}(u)=R_{k}(u) \bmod 1 .
$$

This set is the union of at most two intervals. Further, the sum of the measures of the sets $\tilde{R}_{k}(u)$ is 1 . To prove this, we first consider the case when $k$ is even. Write $k=2 k_{0}$. By Lemma 3.1, if $0 \leq u<q_{k-1}$, then the measure of $R_{k}(u)$

$$
\frac{1}{\varphi^{k_{0}}}-\frac{m-\sqrt{d}}{2 \varphi^{k_{0}}}=\frac{2-m+\sqrt{d}}{2 \varphi^{k_{0}}} .
$$

Further, the measure of $\tilde{R}_{k}(u)$ is equal to the measure of $R_{k}(u)$ as

$$
\frac{1}{\varphi^{k_{0}}}-\frac{m-\sqrt{d}}{2 \varphi^{k_{0}}}<1 .
$$

Similarly, if $q_{k-1} \leq u<q_{k}$, then the measure of $\tilde{R}_{k}(u)$ is

$$
\begin{aligned}
\frac{1}{\varphi^{k_{0}+1}}-\frac{m-\sqrt{d}}{2 \varphi^{k_{0}}} & =\frac{1}{2 \varphi^{k_{0}}}\left(2 \varphi^{-1}-m+\sqrt{d}\right) \\
& =\frac{1}{2 \varphi^{k_{0}}}(m+2-\sqrt{d}-m+\sqrt{d}) \\
& =\frac{1}{\varphi^{k_{0}}} .
\end{aligned}
$$




\section{BASE- $q$ AND OSTROWSKI SUM-OF-DIGITS FUNCTIONS}

Hence, the sum of measures of the sets $\tilde{R}_{k}(u)$ is

$$
\begin{aligned}
\frac{2-m+\sqrt{d}}{2 \varphi^{k_{0}}} q_{k-1}+\frac{1}{\varphi^{k_{0}}}\left(q_{k}-q_{k-1}\right) \\
=\frac{2-m+\sqrt{d}}{2 \varphi^{k_{0}}} q_{k-1}+\frac{1}{\varphi^{k_{0}}}\left((m-1) q_{k-1}+q_{k-2}\right) \\
=\frac{m+\sqrt{d}}{2 \varphi^{k_{0}}} q_{k-1}+\frac{1}{\varphi^{k_{0}}} q_{k-2} \\
=\frac{m+\sqrt{d}}{2 \varphi^{k_{0}}}\left(\frac{1}{\sqrt{d}} \varphi^{k_{0}}-\frac{1}{\sqrt{d}} \varphi^{-k_{0}}\right) \\
+\frac{1}{\varphi^{k_{0}}}\left(\frac{m+\sqrt{d}}{2 \sqrt{d}} \varphi^{k_{0}-1}-\frac{m-\sqrt{d}}{2 \sqrt{d}} \varphi^{-\left(k_{0}-1\right)}\right) \\
=\frac{m+\sqrt{d}}{2 \sqrt{d}}\left(1+\varphi^{-1}\right) \\
\quad-\frac{1}{\varphi^{2 k_{0}}}\left(\frac{m+\sqrt{d}}{2 \sqrt{d}}+\frac{m-\sqrt{d}}{2 \sqrt{d}} \varphi\right) \\
=1-0=1 .
\end{aligned}
$$

A similar calculation shows that the sum of measures also equals one when $k$ is odd. Now we show that the sets $\tilde{R}_{k}(u)$ cover the interval $[0,1)$. If not, pick

$$
x \in[0,1) \backslash \bigcup_{0 \leq u<q_{k}} \tilde{R}_{k}(u) .
$$

Then, there exists $\epsilon>0$ such that the sets $[x, x+\epsilon]$ and $\bigcup \tilde{R}_{k}(u)$ are disjoint. Since $\varphi$ is irrational, the sequence $\left(\left\{p_{k}(n)\right\}\right)$ is dense in $[0,1)$ by Kronecker's theorem (see, for example, [2, Theorem 7.7]). Hence there is an integer $n_{0}$ such that $\left\{p_{k}\left(n_{0}\right)\right\} \in[x, x+\epsilon]$. Therefore

$$
\left\{p_{k}\left(n_{0}\right)\right\} \notin \bigcup_{0 \leq u<q_{k}} \tilde{R}_{k}(u) .
$$

This contradicts Lemma 3.1. Thus the interval $[0,1)$ is the union of the sets $\tilde{R}_{k}(u)$.

Finally, we show that these sets are disjoint. If not, there exist $x, v, w$ with $v \neq w$, such that

$$
x \in \tilde{R}_{k}(v) \cap \tilde{R}_{k}(w) .
$$

Then there is an $\epsilon>0$ such that

$$
\lambda\left(\tilde{R}_{k}(v) \cap \tilde{R}_{k}(w)\right) \geq \epsilon .
$$


Thus

$$
\begin{aligned}
1 & =\lambda\left(\bigcup_{0 \leq u<q_{k}} \tilde{R}_{k}(u)\right) \\
& =\lambda\left(\left(\tilde{R}_{k}(v) \backslash\left(\tilde{R}_{k}(v) \cap \tilde{R}_{k}(w)\right)\right) \cup \bigcup_{u \neq v} \tilde{R}_{k}(u)\right) \\
& \leq \sum_{0 \leq u<q_{k}} \lambda\left(\tilde{R}_{k}(u)\right)-\epsilon=1-\epsilon,
\end{aligned}
$$

which is a contradiction. Therefore the sets $\tilde{R}_{k}(u)$ must be disjoint.

As an immediate consequence of Lemmas 3.1 and 3.2 we get

Corollary 3.3. Let $n \geq 0, k \geq 2$ and $0 \leq u<q_{k}$. Then

$$
t_{\alpha}(n ; k)=u
$$

if and only if

$$
(-1)^{k} n \varphi \in R_{k}(u)+\mathbb{Z}
$$

We now present an inversion formula as in (7) for the function $e\left(\gamma S_{\alpha, k}(n)\right)$.

Lemma 3.4. Let $\gamma \in \mathbb{R}$ and $h, n \in \mathbb{Z}$ with $n \geq 0$. Let $H, k$ be positive integers with $k \geq 2$. Define

$$
\begin{aligned}
& M_{k}^{(1)}(h, \gamma)=\sum_{0 \leq u<q_{k-1}} e\left(\gamma S_{\alpha}(u)-h p_{k}(u)\right), \\
& M_{k}^{(2)}(h, \gamma)=\sum_{q_{k-1} \leq u<q_{k}} e\left(\gamma S_{\alpha}(u)-h p_{k}(u)\right),
\end{aligned}
$$

where $p_{k}(u)$ is as defined in Lemma 3.1. For $|h| \leq H$, there exist complex numbers $b_{H}^{(1)}(h), b_{H}^{(2)}(h), c_{H}^{(1)}(h)$ and $c_{H}^{(2)}(h)$ with

$$
\begin{aligned}
& b_{H}^{(1)}(0)=\left\{\begin{array}{lll}
\frac{2-m+\sqrt{d}}{2 \varphi^{k_{0}}} & \text { if } k=2 k_{0}, & k_{0} \in \mathbb{Z}, \\
\frac{1}{\varphi^{k_{0}}} & \text { if } k=2 k_{0}+1, & k_{0} \in \mathbb{Z},
\end{array}\right. \\
& b_{H}^{(2)}(0)=\left\{\begin{array}{lll}
\frac{1}{\varphi^{k_{0}}} & \text { if } k=2 k_{0}, & k_{0} \in \mathbb{Z}, \\
\frac{-m+\sqrt{d}}{2 \varphi^{k_{0}}} & \text { if } k=2 k_{0}+1, & k_{0} \in \mathbb{Z},
\end{array}\right.
\end{aligned}
$$

and for $i=1,2$,

$$
\begin{aligned}
& \left|b_{H}^{(i)}(h)\right| \leq \min \left(b_{H}^{(i)}(0), \frac{1}{|h|}\right) \quad \text { if } h \neq 0, \\
& \left|c_{H}^{(i)}(h)\right| \leq 2
\end{aligned}
$$


such that

$$
\begin{aligned}
e\left(\gamma S_{\alpha, k}(n)\right)= & \sum_{i=1}^{2}\left(\sum_{|h| \leq H} b_{H}^{(i)}(h) e\left(h p_{k}(n)\right) M_{k}^{(i)}(h, \gamma)\right) \\
& +O\left(\frac{1}{H} \sum_{|h| \leq H} c_{H}^{(1)}(h) e\left(h p_{k}(n)\right) \sum_{0 \leq u<q_{k-1}} e\left(-h p_{k}(u)\right)\right) \\
& +O\left(\frac{1}{H} \sum_{|h| \leq H} c_{H}^{(2)}(h) e\left(h p_{k}(n)\right) \sum_{q_{k-1} \leq u<q_{k}} e\left(-h p_{k}(u)\right)\right),
\end{aligned}
$$

where the expressions within the $O$-parentheses are non-negative real numbers.

Proof. Let $u \in\left[0, q_{k}\right)$ be an integer and let

$$
\chi_{u}=\chi_{R_{k}(u)+\mathbb{Z}}
$$

denote the indicator function of $R_{k}(u)+\mathbb{Z}$. Using Corollary 3.3, we get

$$
\begin{aligned}
e\left(\gamma S_{\alpha, k}(n)\right)= & e\left(\gamma S_{\alpha}\left(t_{\alpha}(n ; k)\right)\right) \\
= & \sum_{0 \leq u<q_{k}} e\left(\gamma S_{\alpha}(u)\right) \chi_{u}\left(p_{k}(n)\right) \\
= & \sum_{0 \leq u<q_{k-1}} e\left(\gamma S_{\alpha}(u)\right) \chi_{u}\left(p_{k}(n)\right) \\
& +\sum_{q_{k-1} \leq u<q_{k}} e\left(\gamma S_{\alpha}(u)\right) \chi_{u}\left(p_{k}(n)\right) .
\end{aligned}
$$

Using Vaaler's [22] trigonometric polynomial approximation to the function $\{x\}-1 / 2$, one obtains (see [20, Eqn. (5.7)]),

$$
\chi_{[a, b)+\mathbb{Z}}(x)=\sum_{|h| \leq H} a_{H}^{\prime}(h) e(h(x-b))+O\left(\kappa_{H}(x-b)+\kappa_{H}(x-a)\right),
$$

where

$$
a_{H}^{\prime}(0)=b-a,\left|a_{H}^{\prime}(h)\right| \leq \min \left(b-a, \frac{1}{|h|}\right) \text { if } h \neq 0
$$

and

$$
\kappa_{H}(t)=\frac{1}{2(H+1)} \sum_{|h| \leq H}\left(1-\frac{|h|}{H+1}\right) e(h t)
$$

Further, $\kappa_{H}(t)$ is a non-negative real number for all real numbers $t$. Write

$$
R_{k}(u)=\left[p_{k}(u)+c, p_{k}(u)+d\right),
$$

where the values of $c$ and $d$ can be seen from Lemma 3.1 . 
Then,

$$
\begin{aligned}
& \sum_{0 \leq u<q_{k-1}} e\left(\gamma S_{\alpha}(u)\right) \chi_{u}\left(p_{k}(n)\right) \\
= & \sum_{|h| \leq H} b_{H}^{(1)}(h) e\left(h p_{k}(n)\right) \sum_{0 \leq u<q_{k-1}} e\left(\gamma S_{\alpha}(u)-h p_{k}(u)\right) \\
+ & O\left(\sum_{\beta \in\{c, d\}} \sum_{0 \leq u<q_{k-1}} \kappa_{H}\left(p_{k}(n)-p_{k}(u)-\beta\right)\right)
\end{aligned}
$$

where

$$
b_{H}^{(1)}(h)=a_{H}^{\prime}(h) e(-h d)
$$

Using (10), we get

$$
b_{H}^{(1)}(0)=d-c
$$

and

$$
\left|b_{H}^{(1)}(h)\right| \leq \min \left(b_{H}^{(1)}(0), \frac{1}{|h|}\right) \text { if } h \neq 0 .
$$

Let $\beta \in\{c, d\}$. Since $\kappa_{H}(t)$ is a non-negative real number for all real numbers $t$, we have

$$
\begin{aligned}
& \left|\sum_{0 \leq u<q_{k-1}} \kappa_{H}\left(p_{k}(n)-p_{k}(u)-\beta\right)\right| \\
& \leq \frac{1}{H} \sum_{|h| \leq H}\left(1-\frac{|h|}{H+1}\right) \sum_{0 \leq u<q_{k-1}} e\left(h\left(p_{k}(n)-p_{k}(u)-\beta\right)\right) .
\end{aligned}
$$

Thus, we obtain the term with $i=1$ claimed in the lemma with

$$
c_{H}^{(1)}(h)=\left(1-\frac{|h|}{H+1}\right)(e(-h c)+e(-h d)),
$$

whose absolute value is at most 2 . Similarly, by considering the sum

$$
\sum_{q_{k-1} \leq u<q_{k}} e\left(\gamma S_{\alpha}(u)\right) \chi_{u}\left(p_{k}(n)\right),
$$

we obtain the term with $i=2$.

LEMmA 3.5. Let $\gamma \in \mathbb{R}$ with $\|m \gamma\| \neq 0$. Then there exist $C, \eta>0$ such that for all $\beta \in \mathbb{R}$ and $k \geq 2$, we have

$$
\left|\frac{1}{q_{k}} \sum_{0 \leq u<q_{k}} e\left(\gamma S_{\alpha}(u)+\beta u\right)\right| \leq C e^{-k \eta} .
$$




\section{BASE- $q$ AND OSTROWSKI SUM-OF-DIGITS FUNCTIONS}

Proof. Set

$$
\begin{gathered}
\mu_{k}=\mu_{k}(\gamma, \beta)=\frac{1}{q_{k}} \sum_{0 \leq u<q_{k}} e\left(\gamma S_{\alpha}(u)+\beta u\right), \\
\tilde{M}_{k}=\max \left(\left|\mu_{k}\right|,\left|\mu_{k-1}\right|\right) \quad \text { and } \quad c_{k}=\gamma+\beta q_{k} .
\end{gathered}
$$

By [11, Lemma $4 \&$ p. 333], $\left(\tilde{M}_{k}\right)$ is a decreasing sequence and for $k \geq 1$,

$$
\begin{aligned}
\tilde{M}_{k+2} & \leq \tilde{M}_{k-1}\left(1-\min \left(\frac{1}{12}, \frac{3}{20}\left(1+\frac{1}{a_{k}}-\frac{1}{a_{k+1}}\right) a_{k+1}^{2}\left\|c_{k}\right\|^{2}\right)\right) \\
& \leq \tilde{M}_{k-1}\left(1-\min \left(\frac{1}{12}, \frac{3}{20 m}\left\|c_{k}\right\|^{2}\right)\right)=: \tilde{M}_{k-1} \psi_{k},
\end{aligned}
$$

as $a_{i} \in\{1, m\}$ for $i \geq 1$. Observe that

$$
\begin{aligned}
\left\|a_{k+3} \gamma\right\| & =\left\|a_{k+3} \gamma+\beta\left(a_{k+3} q_{k+2}+q_{k+1}-q_{k+3}\right)\right\| \\
& \leq\left\|c_{k+1}\right\|+a_{k+3}\left\|c_{k+2}\right\|+\left\|c_{k+3}\right\| .
\end{aligned}
$$

Thus

$$
\min (\|\gamma\|,\|m \gamma\|) \leq m \sum_{1 \leq i \leq 3}\left\|c_{k+i}\right\|,
$$

implying that for every $k$, there exists $i \in\{1,2,3\}$ such that

$$
\left\|c_{k+i}\right\| \geq \frac{\min (\|\gamma\|,\|m \gamma\|)}{3 m}
$$

and hence

$$
\begin{aligned}
\psi_{k+i} & \leq 1-\min \left(\frac{1}{12}, \frac{\min (\|\gamma\|,\|m \gamma\|)^{2}}{60 m^{3}}\right) \\
& =1-\frac{\min (\|\gamma\|,\|m \gamma\|)^{2}}{60 m^{3}}=: \psi .
\end{aligned}
$$

Since $\|m \gamma\| \neq 0, \psi<1$. Fix $k$. Then there exists $k_{i} \in\{k-2, k-3, k-4\}$ such that $\psi_{k_{i}} \leq \psi$. Using (11) and the fact that $\left(\tilde{M}_{j}\right)$ is a decreasing sequence, we get

$$
\tilde{M}_{k} \leq \tilde{M}_{k_{i}+2} \leq \tilde{M}_{k_{i}-1} \psi_{k_{i}} \leq \tilde{M}_{k-5} \psi
$$

Applying this repeatedly, we get

$$
\tilde{M}_{k} \leq \tilde{M}_{5} \psi^{k / 5-2}
$$

completing the proof of the lemma. 


\section{Proof of Theorem 1.2}

With all the ingredients in place, the theorem follows as in [20]. We include the details below. Set

$$
\begin{aligned}
R & =\left\lfloor N^{a-\eta a /(2 \ln \varphi)}\right\rfloor, H & =\left\lfloor N^{4 a+\eta a /(2 \ln \varphi)}\right\rfloor, \\
t & =\lfloor a \ln N / \ln q\rfloor, \quad k & =1+\lfloor 2 a \ln N / \ln \varphi\rfloor,
\end{aligned}
$$

where $\eta$ is as in Lemma 3.5, $\varphi$ is as in (41) and $a$ is sufficiently small. Let

$$
\begin{gathered}
g_{q}(n)=e\left(\theta S_{q}(n)\right), g_{q, t}(n)=e\left(\theta S_{q, t}(n)\right), \\
g_{\alpha}(n)=e\left(\gamma S_{\alpha}(n)\right), g_{\alpha, k}(n)=e\left(\gamma S_{\alpha, k}(n)\right)
\end{gathered}
$$

and

$$
g(n)=g_{q}(n) g_{\alpha}(n)
$$

By Lemma 2.3, we get

$$
\left|\sum_{n<N} g(n)\right|^{2} \ll \frac{N}{R} \sum_{|r|<R}\left(1-\frac{|r|}{R}\right)\left|\sum_{0 \leq n, n+r<N} g(n+r) \overline{g(n)}\right| .
$$

Removing the condition $n+r<N$ from the second sum gives an error which is

$$
\begin{aligned}
& \ll \frac{N}{R} \sum_{|r|<R}\left(1-\frac{|r|}{R}\right)\left|\sum_{N \leq n+r<N+R} g(n+r) \overline{g(n)}\right| \\
& \leq \frac{N}{R} \sum_{|r|<R}\left(1-\frac{|r|}{R}\right) \sum_{N \leq n+r<N+R} 1 \\
& \leq \frac{N}{R} \sum_{|r|<R}\left(1-\frac{|r|}{R}\right) R=\frac{N}{R} \sum_{|r|<R}(R-|r|) \ll N R .
\end{aligned}
$$

Thus

$$
\left|\sum_{n<N} g(n)\right|^{2} \ll \frac{N}{R} \sum_{|r|<R}\left(1-\frac{|r|}{R}\right)\left|\sum_{0 \leq n<N} g(n+r) \overline{g(n)}\right|+O(N R) .
$$

We denote by $N^{\prime}$ the largest multiple of $q^{t}$ not exceeding $N$. Restricting the second sum in (12) to $N^{\prime}$ gives an error which is

$$
\ll \frac{N}{R} \sum_{|r|<R}\left(1-\frac{|r|}{R}\right) q^{t}=\frac{N q^{t}}{R^{2}} \sum_{|r|<R}(R-|r|) \ll N q^{t} .
$$


Hence

$$
\begin{aligned}
\left|\sum_{n<N} g(n)\right|^{2} \ll & \frac{N}{R} \sum_{|r|<R}\left(1-\frac{|r|}{R}\right)\left|\sum_{0 \leq n<N^{\prime}} g(n+r) \overline{g(n)}\right| \\
& +O\left(N R+N q^{t}\right) .
\end{aligned}
$$

From the definitions of the functions $g, g_{q}, g_{\alpha}$, we get

$$
\begin{aligned}
g(n+r) \overline{g(n)} & =g_{q}(n+r) g_{\alpha}(n+r) \overline{g_{q}(n)} \overline{g_{\alpha}(n)} \\
& =e\left(\theta S_{q}(n+r)\right) e\left(\gamma S_{\alpha}(n+r)\right) e\left(-\theta S_{q}(n)\right) e\left(-\gamma S_{\alpha}(n)\right) \\
& =e\left(\theta\left(S_{q}(n+r)-S_{q}(n)\right)\right) e\left(\gamma\left(S_{\alpha}(n+r)-S_{\alpha}(n)\right)\right)
\end{aligned}
$$

Therefore for a fixed $r$, we have

$$
\begin{aligned}
\mid & \sum_{0 \leq n<N^{\prime}} g(n+r) \overline{g(n)} \mid \\
= & \sum_{0 \leq n<N^{\prime}} e\left(\theta\left(S_{q}(n+r)-S_{q}(n)\right)\right) e\left(\gamma\left(S_{\alpha}(n+r)-S_{\alpha}(n)\right)\right) \mid \\
\leq & \left.\mid \sum_{0 \leq n<N^{\prime}} e\left(\theta\left(S_{q, t}(n+r)-S_{q, t}(n)\right)\right) e\left(\gamma\left(S_{\alpha, k}(n+r)-S_{\alpha, k}(n)\right)\right)\right) \mid \\
& +\sum_{0 \leq n<N^{\prime}}^{\prime} 1 \\
= & \left|\sum_{0 \leq n<N^{\prime}} g_{q, t}(n+r) \overline{g_{q, t}(n)} g_{\alpha, k}(n+r) \overline{g_{\alpha, k}(n)}\right|+\sum_{0 \leq n<N^{\prime}}^{\prime} 1,
\end{aligned}
$$

where the second sum $\sum^{\prime}$ runs over those integers $n$ for which either

or

$$
e\left(\theta\left(S_{q}(n+r)-S_{q}(n)\right)\right) \neq e\left(\theta\left(S_{q, t}(n+r)-S_{q, t}(n)\right)\right)
$$

$$
e\left(\gamma\left(S_{\alpha}(n+r)-S_{\alpha}(n)\right)\right) \neq e\left(\gamma\left(S_{\alpha, k}(n+r)-S_{\alpha, k}(n)\right)\right)
$$

Substituting in (13), we get 


$$
\begin{aligned}
\left|\sum_{n<N} g(n)\right|^{2} \ll & \frac{N}{R} \sum_{|r|<R}\left(1-\frac{|r|}{R}\right)\left|\sum_{0 \leq n<N^{\prime}} g_{q, t}(n+r) \overline{g_{q, t}(n)} g_{\alpha, k}(n+r) \overline{g_{\alpha, k}(n)}\right| \\
& +\frac{N}{R} \sum_{|r|<R}\left(1-\frac{|r|}{R}\right)\left(\sum_{0 \leq n<N^{\prime}}^{\prime} 1\right)+O\left(N R+N q^{t}\right)
\end{aligned}
$$

Now by Lemma 2.4,

Therefore,

$$
\sum_{0 \leq n<N^{\prime}}^{\prime} 1 \leq\left(\frac{N r}{q^{t}}+r\right)+\frac{N r}{q_{k-1}} .
$$

$$
\begin{aligned}
& \frac{N}{R} \sum_{|r|<R}\left(1-\frac{|r|}{R}\right)\left(\sum_{0 \leq n<N^{\prime}}^{\prime} 1\right) \leq \frac{N}{R} \sum_{|r|<R} \sum_{0 \leq n<N^{\prime}}^{\prime} 1 \\
& \leq \frac{N}{R} \sum_{|r|<R}\left(\frac{N r}{q^{t}}+r+\frac{N r}{q_{k-1}}\right)=\frac{N}{R}\left(\frac{N}{q^{t}}+1+\frac{N}{q_{k-1}}\right) \sum_{|r|<R} r \\
& \ll \frac{N}{R}\left(\frac{N}{q^{t}}+1+\frac{N}{q_{k-1}}\right) R^{2}=\frac{N^{2} R}{q^{t}}+N R+\frac{N^{2} R}{q_{k-1}} .
\end{aligned}
$$

Substituting in (14), we obtain

$$
\begin{array}{r}
\left|\sum_{n<N} g(n)\right|^{2} \ll \frac{N}{R} \sum_{|r|<R}\left(1-\frac{|r|}{R}\right)\left|\sum_{0 \leq n<N^{\prime}} g_{q, t}(n+r) \overline{g_{q, t}(n)} g_{\alpha, k}(n+r) \overline{g_{\alpha, k}(n)}\right| \\
+O\left(N R+N q^{t}+\frac{N^{2} R}{q^{t}}+\frac{N^{2} R}{q_{k-1}}\right) .
\end{array}
$$

From (5), it follows that $\varphi^{(k-1) / 2} \ll q_{k-1}$. Using this and the choice of $R, t, k$, we find that

$$
\begin{aligned}
& N R+N q^{t}+\frac{N^{2} R}{q^{t}}+\frac{N^{2} R}{q_{k-1}} \\
& \ll N^{1+a-\eta a /(2 \ln \varphi)}+N q^{a \ln _{q} N}+\frac{N^{2} N^{a-\eta a /(2 \ln \varphi)}}{q^{a \ln _{q} N}}+\frac{N^{2} N^{a-\eta a /(2 \ln \varphi)}}{\varphi^{a \ln _{\varphi} N}} \\
& \ll N^{1+a}+N^{2-\eta a /(2 \ln \varphi)} .
\end{aligned}
$$


Thus

$$
\begin{array}{r}
\left|\sum_{n<N} g(n)\right|^{2} \ll \frac{N}{R} \sum_{|r|<R}\left(1-\frac{|r|}{R}\right)\left|\sum_{0 \leq n<N^{\prime}} g_{q, t}(n+r) \overline{g_{q, t}(n)} g_{\alpha, k}(n+r) \overline{g_{\alpha, k}(n)}\right| \\
+O\left(N^{1+a}+N^{2-\eta a /(2 \ln \varphi)}\right)
\end{array}
$$

We use the expressions for $g_{q, t}(n+r), g_{q, t}(n), g_{\alpha, k}(n+r), g_{\alpha, k}(n)$ from (7), (8) and Lemma 3.4. In the product $g_{\alpha, k}(n+r) g_{\alpha, k}(n)$, there are sixteen summands of the following three kinds: four products of the main terms in the expressions for $g_{\alpha, k}(n+r)$ and $g_{\alpha, k}(n)$, eight products of the main terms and error terms, four products of the error terms. We now consider these three cases separately.

Case I. (Summands with both factors as main terms.)

Let $h=h_{1}+h_{2}$ and $\ell=\ell_{1}+\ell_{2}$. We need to estimate

$$
\begin{aligned}
& \frac{N}{R^{2}} \sum_{\substack{\ell_{1}, \ell_{2}<q^{t} \\
\left|h_{1}\right|,\left|h_{2}\right| \leq H}} G_{t}\left(\ell_{1}\right) \overline{G_{t}\left(-\ell_{2}\right)} b_{H}^{(i)}\left(h_{1}\right) \overline{b_{H}^{(j)}\left(-h_{2}\right)} M_{k}^{(i)}\left(h_{1}, \gamma\right) \overline{M_{k}^{(j)}\left(-h_{2}, \gamma\right)} \\
& \times \sum_{|r|<R}(R-|r|) e\left(r\left(\frac{\ell_{1}}{q^{t}}+h_{1}(-1)^{k} \varphi\right)\right) \sum_{n<N^{\prime}} e\left(n\left(\frac{\ell}{q^{t}}+(-1)^{k} h \varphi\right)\right) .
\end{aligned}
$$

We first consider the subcase when $h=0$. If $\ell \not \equiv 0\left(\bmod q^{t}\right)$, then

$$
\sum_{n<N^{\prime}} e\left(n \ell q^{-t}\right)=0
$$

as $q^{t} \mid N^{\prime}$. Hence we assume that $\ell \equiv 0\left(\bmod q^{t}\right)$. Therefore, $G_{t}\left(-\ell_{2}\right)=G_{t}\left(\ell_{1}\right)$, implying that

$$
G_{t}\left(\ell_{1}\right) \overline{G_{t}\left(-\ell_{2}\right)}=\left|G_{t}\left(\ell_{1}\right)\right|^{2} .
$$

We will estimate the sum over $n$ trivially. By Lemma 2.1(ii) and (i),

$$
\begin{aligned}
& \sum_{|r|<R}(R-|r|) e\left(r\left(\frac{\ell_{1}}{q^{t}}+h_{1}(-1)^{k} \varphi\right)\right)=\left|\sum_{r<R} e\left(r\left(\frac{\ell_{1}}{q^{t}}+h_{1}(-1)^{k} \varphi\right)\right)\right|^{2} \\
& \leq\left(\min \left(R, \frac{1}{2}\left\|\ell_{1} q^{-t}+h_{1}(-1)^{k} \varphi\right\|^{-1}\right)\right)^{2} \\
& \leq \min \left(R^{2},\left\|\frac{\ell_{1}}{q^{t}}+h_{1}(-1)^{k} \varphi\right\|^{-2}\right) .
\end{aligned}
$$

Next,

$$
\left|M_{k}^{(j)}\left(-h_{2}, \gamma\right)\right| \leq q_{k} \ll q_{k-1}
$$

$$
q_{k} \leq(m+1) q_{k-1} .
$$


By Lemma 3.4,

$$
\left|b_{H}^{(j)}(0)\right| \leq \frac{2}{\varphi^{k_{0}}} \leq \frac{2}{q_{k}}
$$

since $q_{k} \leq \varphi^{k_{0}}$ by (5). Again, by Lemma 3.4 and the fact that $h=h_{1}+h_{2}=0$, we get

Similarly,

$$
\left|b_{H}^{(j)}\left(-h_{2}\right)\right|=\left|b_{H}^{(j)}\left(h_{1}\right)\right| \ll \min \left(\frac{1}{q_{k}}, \frac{1}{\left|h_{1}\right|}\right) \text { if } h_{1} \neq 0 .
$$

implying that

$$
\left|b_{H}^{(i)}\left(h_{1}\right)\right| \ll \frac{1}{q_{k}}
$$

$$
\left|b_{H}^{(i)}\left(h_{1}\right) M_{k}^{(i)}\left(h_{1}, \gamma\right)\right| \ll \frac{1}{q_{k}}\left|\sum_{u} e\left(\gamma S_{\alpha}(u)-h p_{k}(u)\right)\right|,
$$

where the sum either runs over $u<q_{k-1}$ or $q_{k-1} \leq u<q_{k}$. Thus by the triangle inequality

$$
\begin{aligned}
& \left|b_{H}^{(i)}\left(h_{1}\right) M_{k}^{(i)}\left(h_{1}, \gamma\right)\right| \\
& \leq \frac{1}{q_{k}}\left|\sum_{u<q_{k-1}} e\left(\gamma S_{\alpha}(u)-h p_{k}(u)\right)\right|+\frac{1}{q_{k}}\left|\sum_{u<q_{k}} e\left(\gamma S_{\alpha}(u)-h p_{k}(u)\right)\right| \\
& \leq C e^{-(k-1) \eta}+C e^{-k \eta} \ll e^{-(k-1) \eta},
\end{aligned}
$$

where the last step follows from Lemma 3.5 and the inequality $q_{k-1} \leq q_{k}$. Combining the estimates (17)-(20) and (22), we find that the expression in (16) is

$$
\ll \frac{N^{2} q_{k-1}}{e^{\eta(k-1)} R^{2}} \sum_{\ell_{1}<q^{t}}\left|G_{t}\left(\ell_{1}\right)\right|^{2} \sum_{\left|h_{1}\right| \leq H} \min \left(\frac{1}{q_{k}}, \frac{1}{\left|h_{1}\right|}\right) \min \left(R^{2},\left\|\frac{\ell_{1}}{q^{t}}+h_{1}(-1)^{k} \varphi\right\|^{-2}\right) .
$$

Using (9), the above expression is

$$
\ll \frac{N^{2} q_{k-1}}{e^{\eta(k-1)} R^{2}} \sup _{\ell_{1} \in \mathbb{Z}} \sum_{|h| \leq H} \min \left(\frac{1}{q_{k}}, \frac{1}{|h|}\right) \min \left(R^{2},\left\|\ell_{1} q^{-t}+|h| \varphi\right\|^{-2}\right) .
$$

Write

$$
|h|=s q_{k}+\tilde{h}, \quad 0 \leq \tilde{h}<q_{k}
$$

Then

$$
|h|^{-1} \leq\left(s q_{k}\right)^{-1} \text { and } \min \left(1 /\left(s q_{k}\right), 1 / q_{k}\right)=1 /\left(s q_{k}\right) .
$$

Hence the preceeding expression is

$$
\ll \frac{N^{2} q_{k-1}}{e^{\eta(k-1)} R^{2}} \sum_{s \leq H / q_{k}} \frac{1}{s q_{k}} \times \sup _{\ell_{1} \in \mathbb{Z}} \sum_{\tilde{h}<q_{k}} \min \left(R^{2},\left\|\ell_{1} q^{-t}+\left(s q_{k}+\tilde{h}\right) \varphi\right\|^{-2}\right) .
$$




\section{BASE- $q$ AND OSTROWSKI SUM-OF-DIGITS FUNCTIONS}

By Lemma 2.2 and the inequality $\sum_{s \leq x} s^{-1} \ll \ln x$, we obtain the bound

$$
\ll \frac{N^{2}}{e^{\eta(k-1)} R^{2}} \ln H\left(R q_{k}+R^{2} \ln q_{k}\right) \ll \frac{N^{2}}{e^{\eta(k-1)}} \ln H\left(q_{k-1} R^{-1}+\ln q_{k-1}\right) .
$$

By the choice of $H, R, k$ and the fact that $q_{k-1} \leq \varphi^{(k-1) / 2}$ (from (5) ), the contribution in this subcase is

$$
\begin{aligned}
& \ll\left(4 a+\frac{\eta a}{2 \ln \varphi}\right) \frac{N^{2} \ln N}{e^{\eta(2 a \ln N / \ln \varphi)}}\left(\frac{\varphi^{a \ln _{\varphi} N}}{N^{a-\eta a /(2 \ln \varphi)}}+\ln \varphi^{a \ln _{\varphi} N}\right) \\
& =\left(4 a+\frac{\eta a}{2 \ln \varphi}\right) \ln N\left(N^{2-3 \eta a /(2 \ln \varphi)}+a N^{2-2 \eta a / \ln \varphi} \ln N\right) .
\end{aligned}
$$

Next, we consider the case when $h \neq 0$. Since $\varphi$ is badly approximable, there is a constant $c_{1}$ such that for all integers $\ell^{\prime}, h^{\prime}$ with $h^{\prime} \neq 0$,

Thus

$$
\left|\varphi+\frac{\ell^{\prime}}{h^{\prime} q^{t}}\right|>\frac{c_{1}}{\left(h^{\prime} q^{t}\right)^{2}}
$$

$$
\begin{aligned}
\left\|h \varphi+\ell q^{-t}\right\| & =\min _{j \in \mathbb{Z}}\left|h \varphi-j+\ell q^{-t}\right|=\min _{j \in \mathbb{Z}}|h|\left|\varphi-\frac{j q^{t}-\ell}{h q^{t}}\right| \\
& >|h| \frac{c_{1}}{\left(h q_{t}\right)^{2}}=\frac{c_{1}}{|h| q^{2 t}} .
\end{aligned}
$$

We estimate $G_{t}$ and the sum over $r$ trivially. Next, as in (21), $\left|b_{H}^{(i)}\left(h_{1}\right)\right| \ll q_{k}^{-1}$. Further, since $\left|M_{k}^{(i)}\left(h_{1}, \gamma\right)\right| \leq q_{k}$, we get that $\left|b_{H}^{(i)}\left(h_{1}\right) M_{k}^{(i)}\left(h_{1}, \gamma\right)\right| \ll 1$. Similarly,

$$
\left|b_{H}^{(j)}\left(-h_{2}\right) M_{k}^{(j)}\left(-h_{2}, \gamma\right)\right| \ll 1 .
$$

Thus we obtain that the expression in (16) is

$$
\ll N q^{2 t} \sum_{\substack{\left|h_{1}\right|,\left|h_{2}\right| \leq H \\ h_{1}+h_{2} \neq 0}} \sup _{\ell \in \mathbb{Z}}\left|\sum_{n<N^{\prime}} e\left(n\left(\ell q^{-t}+(-1)^{k} h \varphi\right)\right)\right| \text {. }
$$

Let $h \in \mathbb{Z}$ with $1 \leq|h| \leq 2 H$. The number of pairs $\left(h_{1}, h_{2}\right)$ with $(-1)^{k}\left(h_{1}+h_{2}\right)=h$ is $2 H+1-|h|$. Hence the above expression equals

$$
\begin{aligned}
& N q^{2 t} \sum_{1 \leq|h| \leq 2 H}(2 H+1-|h|) \sup _{\ell \in \mathbb{Z}}\left|\sum_{n<N^{\prime}} e\left(n\left(\ell q^{-t}+h \varphi\right)\right)\right| \\
& \ll N q^{2 t} \sum_{1 \leq|h| \leq 2 H}(2 H+1-|h|) \sup _{\ell \in \mathbb{Z}}\left(\min \left(N, \frac{1}{2\left\|\ell q^{-t}+h \varphi\right\|}\right)\right) \\
& \ll N H q^{2 t} \sum_{1 \leq|h| \leq 2 H}|h| q^{2 t} \ll N H^{3} q^{4 t},
\end{aligned}
$$

by Lemma 2.1 and (23). 
Further, by the choice of $H, t$, the contribution in this subcase is

$$
\ll N^{1+12 a+3 \eta a /(2 \ln \varphi)} q^{4 a \ln _{q} N}=N^{1+16 a+3 \eta a /(2 \ln \varphi)} .
$$

Case II. (Summands with exactly one factor as main term.)

Suppose that the main term comes from the expression for $g_{\alpha, t}(n+r)$. (The other case is similar.) Then, we need to estimate

$$
\begin{aligned}
& \frac{N}{R} \sum_{|r|<R}\left(1-\frac{|r|}{R}\right) \mid \sum_{\substack { n<N^{\prime} \\
\begin{subarray}{c}{\ell_{1}, \ell_{2}<q^{t} \\
\left|h_{1}\right| \leq H{ n < N ^ { \prime } \\
\begin{subarray} { c } { \ell _ { 1 } , \ell _ { 2 } < q ^ { t } \\
| h _ { 1 } | \leq H } }\end{subarray}} e\left(\ell_{1}(n+r) q^{-t}+\ell_{2} n q^{-t}+h_{1} p_{k}(n+r)\right) \\
& G_{t}\left(\ell_{1}, \theta\right) \overline{G_{t}\left(-\ell_{2}, \theta\right)} b_{H}^{(i)}\left(h_{1}\right) M_{k}^{(i)}\left(h_{1}, \gamma\right) \times \\
& O\left(\frac{1}{H} \sum_{\left|h_{2}\right| \leq H} c_{H}^{(j)}\left(h_{2}\right) e\left(h_{2} p_{k}(n)\right) \sum_{u} e\left(-h_{2} p_{k}(u)\right)\right) \mid .
\end{aligned}
$$

Recall that the expression in the error term is a non-negative real number. We use the inequality $\left|M_{k}^{(i)}\left(h_{1}, \gamma\right)\right| \leq q_{k}$, and estimate $G_{t}$ and the sum over $r$ trivially. Using Lemma 3.4, we obtain the following upper bound.

$$
\begin{aligned}
& \frac{N q^{2 t} q_{k}}{H} \sum_{\left|h_{1}\right| \leq H} \min \left(b_{H}^{(i)}(0), \frac{1}{\left|h_{1}\right|}\right) \\
& \quad \times \sum_{\left|h_{2}\right| \leq H}\left|\sum_{u} e\left(-h_{2} p_{k}(u)\right)\right|\left|\sum_{n<N^{\prime}} e\left(h_{2} p_{k}(n)\right)\right| \\
& \ll \frac{N q^{2 t} q_{k} \ln H}{H} \sum_{\left|h_{2}\right| \leq H}\left|\sum_{u} e\left(-h_{2} p_{k}(u)\right)\right|\left|\sum_{n<N^{\prime}} e\left(h_{2} p_{k}(n)\right)\right|,
\end{aligned}
$$

where the last step follows from the inequality $\sum_{s \leq x} s^{-1} \ll \ln x$. By Lemma 2.1, the above expression is

$$
\begin{aligned}
& \ll \frac{N q^{2 t} q_{k} \ln H}{H} \sum_{\left|h_{2}\right| \leq H} \min \left(q_{k}, \frac{1}{\left\|h_{2} \varphi\right\|}\right) \min \left(N, \frac{1}{\left\|h_{2} \varphi\right\|}\right) \\
& \ll \frac{N q^{2 t} q_{k} \ln H}{H} \sum_{\left|h_{2}\right| \leq H} \min \left(q_{k} N, \frac{1}{\left\|h_{2} \varphi\right\|^{2}}\right) \\
& \ll N^{3 / 2} q^{2 t} q_{k}^{3 / 2} \ln H+N^{2} q^{2 t} q_{k}^{2} \frac{(\ln H)^{2}}{H},
\end{aligned}
$$




\section{BASE- $q$ AND OSTROWSKI SUM-OF-DIGITS FUNCTIONS}

where the last inequality follows from Lemma 2.2. By the choice of $H, t, k$ and the fact that $q_{k} \leq \varphi^{k / 2}$, we find that the contribution in this case is

$$
\begin{aligned}
& \ll\left(4 a+\frac{\eta a}{2 \ln \varphi}\right) N^{3 / 2} q^{2 a \ln _{q} N} \varphi^{\left(3 a \ln _{\varphi} N\right) / 2} \ln N \\
& +\left(4 a+\frac{\eta a}{2 \ln \varphi}\right)^{2} N^{2} q^{2 a \ln _{q} N} \varphi^{2 a \ln _{\varphi} N} \frac{(\ln N)^{2}}{N^{4 a+\eta a /(2 \ln \varphi)}} \\
& \leq\left(4 a+\frac{\eta a}{2 \ln \varphi}\right) N^{(3+7 a) / 2} \ln N+\left(4 a+\frac{\eta a}{2 \ln \varphi}\right)^{2} N^{2-\eta a /(2 \ln \varphi)}(\ln N)^{2} .
\end{aligned}
$$

Case III. (Summands with both factors as error terms.)

We need to estimate

$$
\begin{aligned}
& \frac{N}{R} \sum_{|r|<R}\left(1-\frac{|r|}{R}\right) \mid \sum_{n<N^{\prime}} \sum_{\ell_{1}, \ell_{2}<q^{t}} e\left(\ell_{1}(n+r) q^{-t}+\ell_{2} n q^{-t}\right) \\
& G_{t}\left(\ell_{1}, \theta\right) \overline{G_{t}\left(-\ell_{2}, \theta\right)} \\
& \quad \times O\left(\frac{1}{H} \sum_{\left|h_{1}\right| \leq H} c_{H}^{(i)}\left(h_{1}\right) e\left(h_{1} p_{k}(n+r)\right) \sum_{u} e\left(-h_{1} p_{k}(u)\right)\right) \\
& \quad \times O\left(\frac{1}{H} \sum_{\left|h_{2}\right| \leq H} c_{H}^{(j)}\left(h_{2}\right) e\left(h_{2} p_{k}(n)\right) \sum_{u} e\left(-h_{2} p_{k}(u)\right)\right) .
\end{aligned}
$$

We estimate one of the error terms trivially by $q_{k}$. Further, we estimate $G_{t}$ and the sum over $r$ trivially to get

$$
\ll \frac{N q^{2 t} q_{k}}{H} \sum_{\left|h_{2}\right| \leq H}\left|\sum_{u} e\left(-h_{2} p_{k}(u)\right)\right|\left|\sum_{n<N^{\prime}} e\left(h_{2} p_{k}(n)\right)\right| .
$$

This is the product of the expression in (24) with $(\ln H)^{-1}$. Therefore, proceeding as in Case II, we obtain the bound

$$
\begin{aligned}
& \ll N^{3 / 2} q^{2 t} q_{k}^{3 / 2}+N^{2} q^{2 t} q_{k}^{2} \frac{\ln H}{H} \\
& \ll N^{(3+7 a) / 2}+\left(4 a+\frac{\eta a}{2 \ln \varphi}\right) N^{2-\eta a /(2 \ln \varphi)} \ln N .
\end{aligned}
$$

Combining the three cases and (15), we get

$$
\left|\sum_{n<N} g(n)\right|^{2} \ll N^{2-2 \delta} \quad \text { for some } \delta>0 .
$$


Proof of Corollary 1.3.

We first recall an estimate of Gel'fond [13]: If $k_{1}, m_{1}, q$ are positive integers with

$$
m_{1} \geq 2, \quad k_{1}<m_{1} \quad \text { and } \quad \operatorname{gcd}\left(m_{1}, q-1\right)=1,
$$

then there exists $\delta_{1}>0$ such that for every $a \in \mathbb{R}$, we have

$$
\sum_{n<N} e\left(a n+\frac{k_{1}}{m_{1}} S_{q}(n)\right)=O\left(N^{1-\delta_{1}}\right) .
$$

Now, since

$$
\frac{1}{b} \sum_{0 \leq \ell<b} e\left(\frac{a}{b} \ell\right)=1 \text { or } 0
$$

according to whether $b$ divides $a$ or not, we have

$$
\begin{aligned}
& \left|\left\{0 \leq n<N: S_{q}(n) \equiv r_{1}\left(\bmod m_{1}\right), \quad S_{\alpha}(n) \equiv r_{2}\left(\bmod m_{2}\right)\right\}\right| \\
& =\sum_{n<N} \frac{1}{m_{1}} \sum_{0 \leq k_{1}<m_{1}} e\left(k_{1} \frac{S_{q}(n)-r_{1}}{m_{1}}\right) \frac{1}{m_{2}} \sum_{0 \leq k_{2}<m_{2}} e\left(k_{2} \frac{S_{\alpha}(n)-r_{2}}{m_{2}}\right) \\
& =\frac{1}{m_{1} m_{2}} \sum_{\substack{0 \leq k_{1}<m_{1} \\
0 \leq k_{2}<m_{2}}} e\left(-\frac{k_{1} r_{1}}{m_{1}}-\frac{k_{2} r_{2}}{m_{2}}\right) \sum_{n<N} e\left(\frac{k_{1}}{m_{1}} S_{q}(n)+\frac{k_{2}}{m_{2}} S_{\alpha}(n)\right) \\
& =\frac{N}{m_{1} m_{2}}+O\left(\frac{1}{m_{1} m_{2}} \sum_{1 \leq k_{1}<m_{1}}\left|\sum_{n<N} e\left(\frac{k_{1}}{m_{1}} S_{q}(n)\right)\right|\right. \\
& \left.\quad+\frac{1}{m_{1} m_{2}} \sum_{\substack{0 \leq k_{1}<m_{1} \\
1 \leq k_{2}<m_{2}}}\left|\sum_{n<N} e\left(\frac{k_{1}}{m_{1}} S_{q}(n)+\frac{k_{2}}{m_{2}} S_{\alpha}(n)\right)\right|\right) \\
& =\frac{N}{m_{1} m_{2}}+O\left(N^{1-\delta^{\prime}}\right),
\end{aligned}
$$

where the last equality follows from (25) and Theorem 1.2 .

ACKNowledgements. The author thanks the University of Waterloo, Canada for its kind hospitality during the writing of this paper. 


\section{BASE- $q$ AND OSTROWSKI SUM-OF-DIGITS FUNCTIONS}

\section{REFERENCES}

[1] ALlOUChE, J.P.-SHAllit, J.: Automatic Sequences. Cambridge University Press, Cambridge, 2003.

[2] APOStol, T. M.: Modular Functions and Dirichlet Series in Number Theory. In: Graduate Texts in Mathematics Vol. 41 (2nd ed.), Springer-Verlag, Berlin, 1990.

[3] BARAT, G.-LIARDET, P.: Dynamical systems originated in the Ostrowski alphaexpansion, Ann. Univ. Sci. Budapest. Sect. Comput. 24, (2004), 133-184.

[4] BASSILY, N. L.-KÁTAI, I.: Distribution of the values of q-additive functions on polynomial sequences, Acta Math. Hungar. 68 (1995), no. 4, 353-361.

[5] BEREND, D.-KOLESNIK, G.: Joint distribution of completely q-additive functions in residue classes, J. Number Theory 160, (2016), 716-738,

[6] BERTHÉ, V.: Autour du système de numération d'Ostrowski, Journées Montoises d'Informatique Théorique (Marne-la-Vallée, 2000), Bull. Belg. Math. Soc. Simon Stevin 8 (2001), no. 2, 209-239. In French, with French summary

[7] BÉSINEAU, J.: Indépendance statistique d'ensembles liés à la fonction "somme des chiffres", Acta Arith. 20 (1972), 401-416. (In French)

[8] BURGER, EB.-CLYDE, D. C.-COLBERT, C. H.-SHIN, G. H.-WANG, Z.: A generalization of a theorem of Lekkerkerker to Ostrowski's decomposition of natural numbers, Acta Arith. 153, (2012), no. 3, 217-249.

[9] BUSH, L. E.: An asymptotic formula for the average sum of the digits of integers, Amer. Math. Monthly 47 (1940), 154-156.

[10] COQUET, J.-RHIN, G.-TOFFIN, P.: Représentations des entiers naturels et indépendance statistique II, Ann. Inst. Fourier (Grenoble), 31 (1981), no. 1, ix, 1-15. (In French)

[11] COQUET, J.-RHIN, G.-TOFFIN, P.: Fourier-Bohr spectrum of sequences related to continued fractions, J. Number Theory 17 (1983), no. 3, 327-336.

[12] DARTYGe, C.-TEnEnBAuM, G.: Sommes des chiffres de multiples d'entiers, Ann. Inst. Fourier (Grenoble), 55 (2005), no. 7, 2423-2474. (In French, with English and French summaries)

[13] GEL'FOND, A. O.: Sur les nombres qui ont des propriétés additives et multiplicatives données, Acta Arith. 13 (1967/1968), 259-265. (In French)

[14] GRAhAM, S. W.-KOLESNIK, G.: Van der Corput's Method of Exponential Sums. London Mathematical Society Lecture Note Series Vol. 126, In: Cambridge University Press, Cambridge, 1991.

[15] KÁtAi, I.-MOGYORÓDI, J.: On the distribution of digits, Publ. Math. Debrecen 15 (1968), 57-68.

[16] KIM, D.-H.: On the joint distribution of $q$-additive functions in residue classes, J. Number Theory 74 (1999), no. 2, 307-336.

[17] KOROBOV, N. M.: Exponential Sums and their Applications. In: Mathematics and its Applications (Soviet Series) Vol. 80, Translated from the 1989 Russian original by Yu. N. Shakhov, Kluwer Academic Publishers Group, Dordrecht, 1992. 


\section{DIVYUM SHARMA}

[18] MAUDUIT, CH.-RIVAT, J.: Sur un problème de Gelfond: la somme des chiffres des nombres premiers, Ann. of Math.(2) 171 (2010), no. 3, 1591-1646. (In: French with English and French summaries)

[19] OSTROWSKI, A.: Bemerkungen zur Theorie der Diophantischen Approximationen, Abh. Math. Sem. Univ. Hamburg 1 (1922), no. 1, 77-98. In German

[20] SPIEGELHOFER, L.: Correlations for Numeration Systems, PhD Thesis, Vienna, 2014.

[21] Pseudorandomness of the Ostrowski sum-of-digits function, arXiv:1611.03043 [math.NT].

[22] VAALER, J.D.: Some extremal functions in Fourier analysis, Bull. Amer. Math. Soc. (N.S.), 12, (1985), no. 2, 183-216.

[23] ZECKENDORF, E.: Représentation des nombres naturels par une somme de nombres de Fibonacci ou de nombres de Lucas, Bull. Soc. Roy. Sci. Liège 41 (1972), 179-182. (In French with English summary)

Received November 16, 2017

Accepted December 11, 2018
Divyum Sharma

Department of Pure Mathematics

University of Waterloo

Ontario, CANADA

Current Address:

Department of Mathematics

Birla Institute of Technology and Science, Pilani

Vidya Vihar, Rajasthan INDIA

E-mail: divyum.sharma@pilani.bits-pilani.ac.in 